\title{
Visualization of plasmonic couplings using ultrafast electron microscopy
}

Haihua Liu ${ }^{1, *}$, Thomas E. Gage ${ }^{1}$, Prem Singh ${ }^{2}$, Amit Jaiswal ${ }^{2}$, Richard D. Schaller ${ }^{1}$, Jau Tang ${ }^{3}$, Sang Tae Park ${ }^{4}$, Stephen K. Gray ${ }^{1}$, Ilke Arslan ${ }^{1, *}$

1. Center for Nanoscale Materials, Argonne National Laboratory, Lemont IL 60439, USA

2. School of Basic Sciences, Indian Institute of Technology Mandi, Kamand, Mandi 175005, Himachal Pradesh, India.

3. The Institute of Technological Sciences, Wuhan University, Wuhan 430072, China

4. IDES, Inc. (a JEOL company), Pleasanton, CA 94588, USA.

* Corresponding authors: haihua.liu@ anl.gov or arslan@ anl.gov

\section{Simulation:}

The electromagnetic simulations were carried out by using the discrete-dipole-approximation code, DDA. ${ }^{1}$ DDA is a general method to calculate the scattering and absorption of the electromagnetic wave by arbitrary geometry particles. The Au nanocapsule and graphene are divided into point dipole arrays. The mesh grid size used in the simulation here was $2 \mathrm{~nm}$. The hollow Au nanocapsule has one dimension of $60 \times 360 \mathrm{~nm}$ with a $10 \mathrm{~nm}$ thick shell and an $\mathrm{Au}$ core of $40 \times 60 \mathrm{~nm}$. The graphene sheet in Fig. $4 \mathrm{a}$ had $4 \mathrm{~nm}$ thickness. The graphene step of $4 \mathrm{~nm}$ high is overlapped on a graphene sheet of $4 \mathrm{~nm}$ thickness in Fig. 4b. The distance from the GNC tip/edge to the graphene substrate sheet edge was set as $200 \mathrm{~nm}$. The near field is calculated based on the method in a prior published paper. ${ }^{2}$ For the hybrid model of Fig. 4a, the graphene thickness was changed from $2 \mathrm{~nm}$ to $16 \mathrm{~nm}$. The plasmonic field of single GNC without graphene substrate was also simulated for comparison. For the hybrid model of Fig. 4b, the tipstep distance was changed from $-120 \mathrm{~nm}$ to $200 \mathrm{~nm}$ in the simulation. The optical constants of $\mathrm{Au}$ and graphene are from published references. ${ }^{3,4}$

Fig. S1 shows the discrete dipole approximation (DDA) simulation results for a $60 \times 360 \mathrm{~nm}$ gold nanocapsule (GNC) with a $10 \mathrm{~nm}$ thick shell and a $40 \times 60 \mathrm{~nm}$ core under $1030 \mathrm{~nm}$ optical excitation. The field intensities of total field $|E|^{2}$ and component fields $\left(\left|E_{x}\right|^{2},\left|E_{y}\right|^{2},\left|E_{z}\right|^{2}\right)$ are mapped by false color contrast at the plane of $\mathrm{z}=52 \mathrm{~nm}$ (above the bottom surface of the GNC) in Figs. S1a-d, and at the plane of $z=30 \mathrm{~nm}$ (above the bottom surface of the GNC) in Figs. S1e-h under light polarization parallel to the longitudinal axis, respectively. Figs. S1i-1 and Fig. S1m-p are plasmonic field mapping at $\mathrm{z}=52 \mathrm{~nm}$ and $\mathrm{z}=30 \mathrm{~nm}$ under light polarization perpendicular to 
the longitudinal axis. The light polarization orientation is indicated by the red arrow. As seen from the maximum intensity labelled on the simulation images, the longitudinal polarization excitation has much stronger plasmonic field than the transverse polarization excitation as a factor of about 30 under the same wavelength optical excitation, which is in agreement with the intensity difference between the photon-induced near field electron microscopy (PINEM) images in Figs. 2c and 2d. The mean and maximum intensities of $|E|^{2},\left|E_{x}\right|^{2},\left|E_{y}\right|^{2},\left|E_{z}\right|^{2}$ at the tip of the GNC are plotted in Figs. S1q and S1r under longitudinal light polarization excitation. The experimental PINEM image intensity is proportional to the square of the PINEM field $F_{\mathrm{z}}$, which is the Fourier transform of the z-component plasmonic field $E_{z}$ at the spatial frequency of $\Delta k_{\mathrm{e}}=\omega_{\mathrm{p}} / v_{\mathrm{e}}{ }^{5}$

$$
F_{\mathrm{z}}\left(\Delta k_{\mathrm{e}}\right)=\int_{-\infty}^{+\infty} d z^{\prime \prime} E_{\mathrm{z}}\left(z^{\prime \prime}, 0\right) e^{-i \Delta k \mathrm{e} z^{\prime \prime}}
$$

where $\omega_{\mathrm{p}}$ is the angular frequency of the incident light, $v_{\mathrm{e}}$ is the group velocity of the traveling electrons. For light with $k_{\mathrm{p}}=\Delta k_{\mathrm{e}}$, the phase velocity is reduced to $v_{\mathrm{p}}=v_{\mathrm{e}}$ to satisfy the energy/momentum conservation condition which makes it possible for photon-electron interaction in the scattered plasmonic field of the GMC. Fig. S1s is the calculated PINEM field intensity $\left|\mathrm{F}_{\mathrm{z}}\right|^{2}$ mapping of the GNC under longitudinal light excitation.

Fig. S2a shows one transmission electron microscopy (TEM) bright field image of a GNC $(60 \times 340 \mathrm{~nm})$ located on a graphene step island. A GNC tip is overlapping on the graphene step edge, and the other tip is $\sim 30 \mathrm{~nm}$ from the closest step edge. The false color contrast in Fig. S2a aids in visualizing the graphene steps. Figs. S2b and S2c display two PINEM images of the GNC under $1030 \mathrm{~nm}$ optical excitation with different light polarizations. In Fig.S2b, the GNC was excited under longitudinal optical excitation with higher PINEM image intensity at the right tip than the left tip. The observed asymmetrical PINEM image intensities were related to the different tip-step distances at two tips. Although the closest distance between the GNC left tip to the graphene step is about $30 \mathrm{~nm}$, the actual distance between the tip and the step along the longitudinal axis (light polarization orientation) is about $160 \mathrm{~nm}$. The transverse plasmon mode of the GNC was not excited effectively by $1030 \mathrm{~nm}$ optical pulse, as seen in Fig. S2c. Fig. S2d show two PINEM spectra obtained at delay time of $\mathrm{t}=0 \mathrm{ps}$ at the left and right tips, which are normalized to each zero-loss peak. The insert in Fig. S2d is the normalized peaks intensity. The spectrum at the right tip has stronger peaks intensity than that at the left tip. Fig. S2e plots the 
line intensity profile along the longitudinal axis of the GNC from left to right. The PINEM image intensity ratio of right/left GNC tips here is about 2.2. Fig. S2b, S2d and S2e all imply that the local z-component electromagnetic field at the right tip is stronger than that at the left tip caused by the coupling between the graphene step and the GNC. We recorded the time-resolved PINEM images by changing the delay time between the pump beam and the probe beam. The average intensity at the left tip and right tip in the time-resolved PINEM images is plotted in Fig. S2f. The FWHM of the time-resolved image intensity is about $843 \mathrm{fs}$ at the right tip and $786 \mathrm{fs}$ at the left tip. The PINEM image has the maximum intensity at the delay time of $\mathrm{t}=0 \mathrm{ps}$ and then decayed quickly within a few hundred femtoseconds.

Figs. S3 plot the max field intensity within the rectangle boxes around the tips at different $\mathrm{z}$ height for the symmetrical model of Fig. 4a and asymmetrical model of Fig. 4b, in which the max field intensity occur at the interface between the graphene substrate and the GNC with strong field confinement. As seen in Figs. S3b, the field intensity at the left tip is higher than that at the right tip at all the z-height for the model of Fig. 4b. The interface between the GNC and the graphene has the highest field intensity, which means that the field is highly confined at the interface gap. We chose the $\mathrm{z}$ plane of $52 \mathrm{~nm}$ above the top surface of the graphene step as one reference to compare the field intensities between two tips in Figs. 4c, 4d, 4g and 4h. For the hybrid model similar as Fig. 4b, different graphene substrate thickness and step height are simulated for the tip-step distance series like Fig. 4d. The max field intensity of total field $|\mathrm{E}|^{2}$ and z-component field $\left|E_{z}\right|^{2}$ and their relative intensity ratio at the left and right tips are plotted against with the tip-step distance changing from $-120 \mathrm{~nm}$ to $200 \mathrm{~nm}$ in Figs. S3, $2 \mathrm{~nm}+2 \mathrm{~nm}$ (c), $2 \mathrm{~nm}+4 \mathrm{~nm}(\mathrm{~d}), 4 \mathrm{~nm}+2 \mathrm{~nm}(\mathrm{~d})$, and $4 \mathrm{~nm}+8 \mathrm{~nm}(\mathrm{~d})$. The max field intensities for $|\mathrm{E}|^{2}$ and $\left|\mathrm{E}_{z}\right|^{2}$ in Fig. S3c-d are all extracted at the same GNC height of $52 \mathrm{~nm}$ above the top surface of the graphene step. In Fig. S3c and S3d, the field intensity and the corresponding intensity ratio have oscillation phenomenon. For the graphene substate thickness $>=4 \mathrm{~nm}$ in Fig. S3e and S3f, the field intensity at the left tip is higher than that at the right tip. The above simulation results indicate that the graphene step effect on the plasmonic field of the GNC is not only tip-step distance dependent but also graphene substrate thickness and step height dependent.

Fig. S4 show the simulated angle effect between the GNC and the graphene step. Fig. S4a and S4b show two models for the GNC on graphene step with the angle of $90^{\circ}$ and $60^{\circ}$ between the 
long axis of the GNC and the graphene step edge. Fig. S4c and S4d are the z-component plasmonic field intensities $\left|E_{z}\right|^{2}$ distributions for the angle of $90^{\circ}$ and $60^{\circ}$, which both show the field intensities are higher at the left tip than that at the right tip. Fig. S4e plots the field intensities $\left|\mathrm{E}_{\mathrm{z}}\right|^{2}$ at the left tip and right tip and the ratio between the left and right against the angle changing from $20^{\circ}$ to $90^{\circ}$. With the angle increasing from $20^{\circ}$ to $90^{\circ}$, the ratio of $\mathrm{L} / \mathrm{R}$ increases firstly and then decreases. Within the angle range of $20^{\circ}$ to $90^{\circ}$, the maximum field intensities $\left|\mathrm{E}_{\mathrm{z}}\right|^{2}$ at the left tip close to the graphene step edge are higher than that at the right tip.

In Fig. S5a, there are graphene steps separated by a distance changing from 200 to $300 \mathrm{~nm}$. Figs. $\mathrm{S} 5 \mathrm{~b}$ and S5c are PINEM images at delay time of $\mathrm{t}=0 \mathrm{ps}$ under $1030 \mathrm{~nm}$ excitation with different light polarization. The color lines indicate the graphene step in Fig. S5a. The PINEM image contrast appeared on the steps when the light polarization is perpendicular to the steps or has a large perpendicular component on the steps, which indicated that there were oscillating charges interacting with travelling electrons under light excitation. In Fig. S5d, there is one irregular hole (size $835 \times 1200 \mathrm{~nm}$ ) on the graphene film. Figs. S5e and S5f shows the localized plasmonic field near the hole edge under $1030 \mathrm{~nm}$ excitation with different polarization. The electromagnetic field is confined within the distance of about $200 \mathrm{~nm}$ from the graphene edge. Compared with Figs. S5b and S5c, the plasmon field mainly extends into the vacuum in Figs. S5e and S5f. The near field near the graphene step is localized surface plasmon resonance due to the collective charge oscillating produced by optical excitation. ${ }^{6}$ In Fig. $2 \mathrm{c}$ we didn't observe the graphene surface plasmons due to the absence of a graphene step or edge, which lacks momentum matching between the incident electrons and the photons. Here the PINEM image contrast observed at the graphene steps or edges is caused by the localized surface plasmons of the graphene under 1030 optical excitation at nanoscale.

\section{Au nanocapsule growth process:}

\section{Materials preparation:}

Following chemicals were used for the synthesis of Au nanocapsules: Gold(III) chloride trihydrate $\left(\mathrm{HAuCl}_{4} 3 \mathrm{H}_{2} \mathrm{O}\right)$, citric acid, sodium borohydride $\left(\mathrm{NaBH}_{4}\right)$, L-ascorbic acid, hexadecyltrimethylammonium chloride (CTAC), and benzyldimethylhexadecylammonium chloride (BDAC). CTAC was procured from TCI and the rest of the chemicals were procured from Sigma-Aldrich. All synthesis experiments were performed using nanopure water (resistivity 18.2 
$\mathrm{M} \Omega \cdot \mathrm{cm}$ ) along with aqua-regia cleaned glass wares. Three step seed-mediated method was used for the preparation of Au nanocapsule. ${ }^{7}$ The detailed sample growth process is described in the supporting materials. The TEM specimen used in the UEM experiments was prepared by dropping Au nanocapsule solution on graphene TEM grids (Graphene Supermarket).

Instrumentation: Magnetic stirrer IKA (C-MAG HS7) was used for stirring and maintaining the temperature during synthesis process. The extinction spectra were recorded using Lambda 750 UV/VIS/NIR spectrophotometer (Perkin Elmer) and UV-1800 spectrophotometer (Shimadzu, Japan). The morphological study of the synthesized nanostructures was performed using transmission electron microscopy (TEM, FP 5022/22-Tecnai G2 20 STWIN, FEI).

Synthesis of Au nanocapsule: 3 step seed-mediated method was used for the preparation of $\mathrm{Au}$ nanocapsule using a previously reported method ${ }^{3}$ with few modifications. In the first step, seed solution for the synthesis of Au beads was prepared by adding $0.25 \mathrm{~mL}$ of ice-cold sodium borohydride solution $(25 \mathrm{mM})$ into $10 \mathrm{~mL}$ of solution containing CTAC $(50 \mathrm{mM}), \mathrm{HAuCl}_{4}(0.18$ $\mathrm{mM})$ and citric acid $(5 \mathrm{mM})$. After few minutes of vigorous stirring at room temperature, the vial was transferred in an oil bath under mild stirring at $90^{\circ} \mathrm{C}$ for 400 minutes. For the further growth, $0.10 \mathrm{~mL}$ of seed solution was added under vigorous stirring to a growth solution containing BDAC $(10 \mathrm{~mL}, 50 \mathrm{mM}), \mathrm{HAuCl}_{4} .3 \mathrm{H}_{2} \mathrm{O}(0.1 \mathrm{~mL}, 35.6 \mathrm{mM})$ and ascorbic acid $(0.075 \mathrm{~mL}, 100 \mathrm{mM})$ at 30 ${ }^{\circ} \mathrm{C}$. The resulting mixture was left undisturbed at $30{ }^{\circ} \mathrm{C}$ for 30 minutes. After 30 minutes, the assynthesized Au beads were washed twice using CTAC $(80 \mathrm{mM})$. In the $2^{\text {nd }}$ growth step, $2 \mathrm{~mL}$ of processed $\mathrm{Au}$ beads were added in a growth mixture having $2.5 \mathrm{~mL}$ of CTAC $(50 \mathrm{mM})$, desired amount of $\mathrm{AgNO}_{3}(2 \mathrm{mM})$ and $0.8 \mathrm{~mL}$ of ascorbic acid $(0.1 \mathrm{M})$. The following mixture was stirred at $1000 \mathrm{rpm}$ at $65{ }^{\circ} \mathrm{C}$ for $30 \mathrm{~min}$. In the last step, Au nanocapsules were synthesized by using a galvanic exchange reaction where silver shell of Au beads@Ag nanorods were transformed into hollow and porous shell of Au. In details, the as synthesized Au beads@ Ag rods were centrifuged and dispersed in a CTAC solution $(50 \mathrm{mM})$, followed by heating at $120{ }^{\circ} \mathrm{C}$. Under stirring condition, the aqueous solution of $\mathrm{HAuCl}_{4} .3 \mathrm{H}_{2} \mathrm{O}(0.5 \mathrm{mM})$ was added at a rate of $0.25 \mathrm{~mL} / \mathrm{min}$ until the color of the solution turned to greyish blue. 


\section{Reference:}

1. Yurkin, M. A., Hoekstra, A. G. The discrete-dipole-approximation code ADDA: Capabilities and known limitations. J. Quan. Spec. \& Rad. 112, 2234-2247 (2011).

2. D'Agostino, S. et al. Enhanced fluorescence by metal nanospheres on metal substrates. Optics Lett. 34, 2381-2383 (2009).

3. Johnson, P. B. and Christy, R. W. Optical constants of the noble metals, Phys. Rev. B 6, 43704379 (1972).

4. Weber, J. W., Calado, V. E., and Van De Sanden, M. C. M. Optical constants of graphene measured by spectroscopic ellipsometry, Appl. Phys. Lett. 97, 091904 (2010).

5. Part, S. T., Lin, M. M., Zewail, A. H. Photon-induced near-field electron microscopy (PINEM): theoretical and experimental. New J. Phys. 12, 123028 (2010).

6. Park, S. T., Yurtsever, A., Baskin, J. S., Zewail, A. H. Graphene-layered steps and their fields visualized by 4D electron microscopy. Proc. Natl. Acad. Sci. U.S.A 110(23), 9277-9282 (2013). 7. Singh, P., König, T.A.F., Jaiswal, A. NIR-active plasmonic gold nanocapsules synthesized using thermally induced seed twinning for surface-enhanced raman scattering applications. ACS Appl. Mater. Interfaces 10 (45), 39380-39390 (2018). 


\section{Figures}

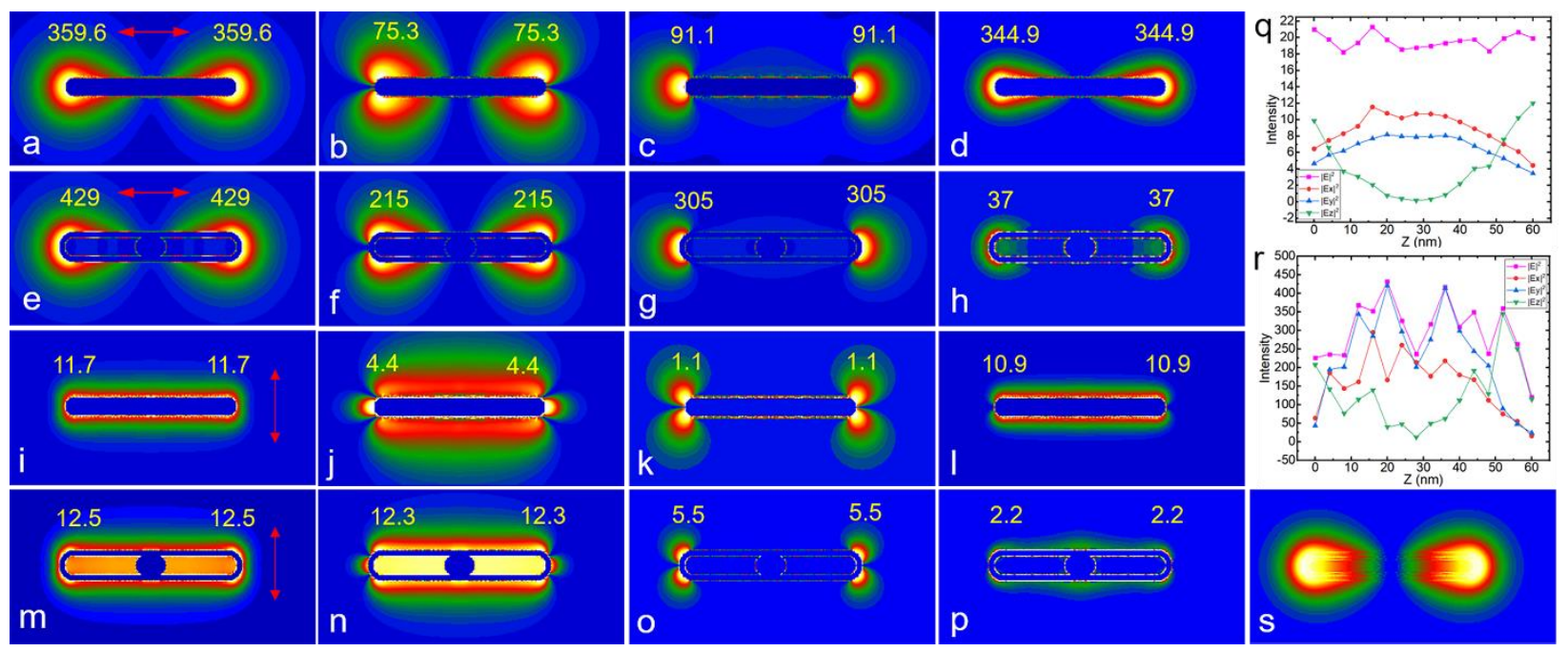

Figure S1 Plasmonic field simulation of the GNC. The $60 \times 360 \mathrm{~nm}$ hollow GNC has an Au shell of $10 \mathrm{~nm}$ thickness and a solid Au core of dimensions $40 \times 60 \mathrm{~nm}$. a-d and e-h are the field intensities mapping of the total field $|\mathrm{E}|^{2}$ and field components $\left|\mathrm{E}_{\mathrm{x}}\right|^{2},\left|\mathrm{E}_{\mathrm{y}}\right|^{2},\left|\mathrm{E}_{\mathrm{z}}\right|^{2}$ with light polarization parallel to the longitudinal axis at $\mathrm{z}=52 \mathrm{~nm}$ and $\mathrm{z}=30 \mathrm{~nm}$ (above the bottom surface of the GNC), respectively. $\mathbf{i}-\mathbf{l}$ and $\mathbf{m}-\mathbf{p}$ are the field intensity mapping of the total field $|\mathrm{E}|^{2}$ and field components $\left|E_{x}\right|^{2},\left|E_{y}\right|^{2},\left|E_{z}\right|^{2}$ with light polarization perpendicular to the longitudinal axis at $\mathrm{z}=52 \mathrm{~nm}$ and $\mathrm{z}=30 \mathrm{~nm}$ (above the bottom surface of the GNC), respectively. $\mathbf{q}$ and $\mathbf{r}$ are the $\mathrm{z}-$ dependence of mean and maximum field intensity, respectively. s shows PINEM field intensity $\left|\mathrm{F}_{\mathrm{z}}\right|^{2}$ mapping. 

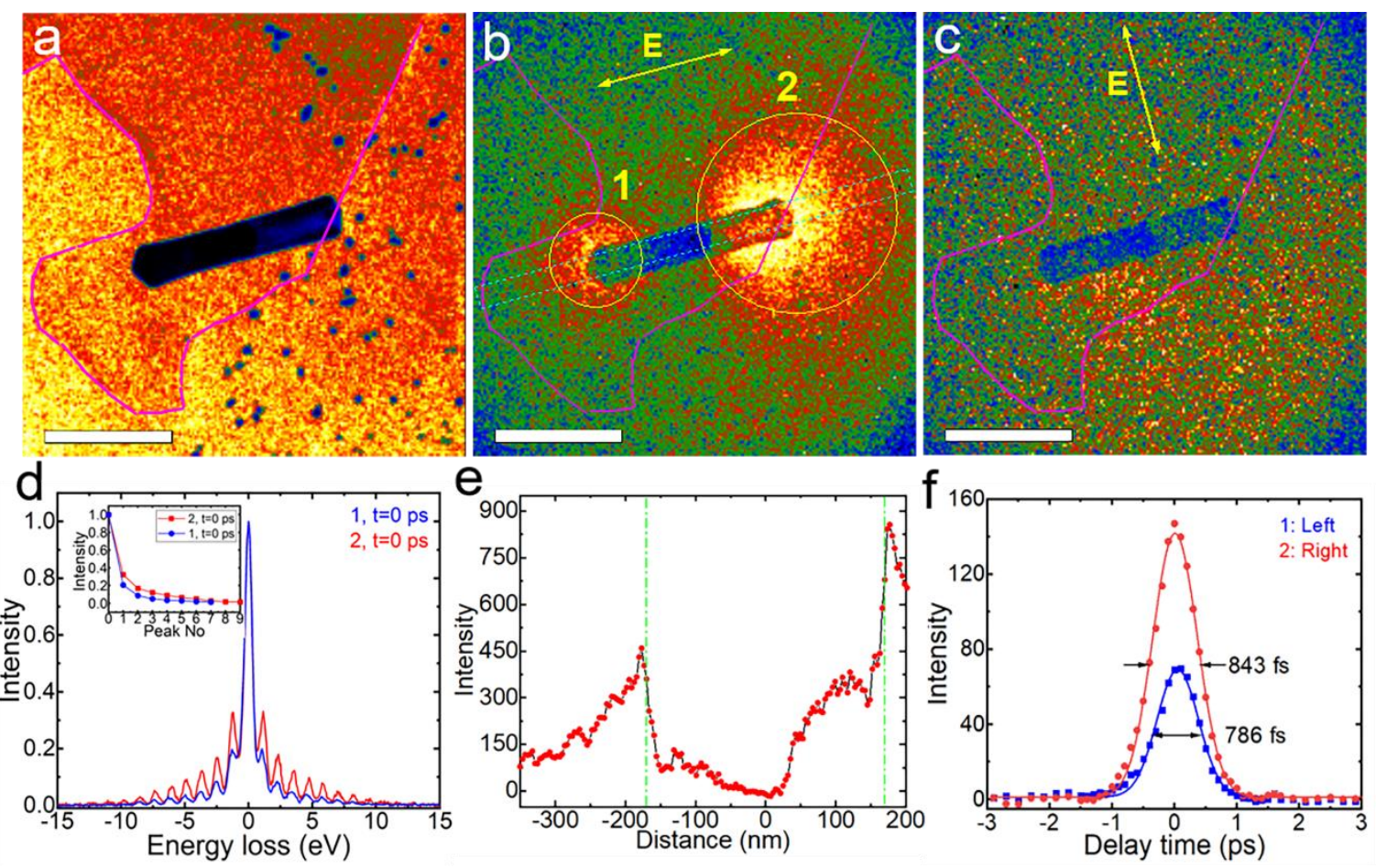

Figure S2 Plasmonic field of a single GNC located on one graphene step island. a, TEM bright field image of a GNC on the graphene step island. $\mathbf{b}$ and $\mathbf{c}$ show the PINEM images with light polarizations parallel and perpendicular polarization directions to the longitudinal axis, respectively. d, Two PINEM spectra were obtained at delay time of $\mathrm{t}=0 \mathrm{ps}$ at left and right tips. The insert in $\mathbf{d}$ is the PINEM peaks intensity normalized to zero loss peak. e, The image intensity profile along the line roi of $35 \mathrm{~nm}$ width in $\mathbf{b}$. The green dot-dash lines are at the GNC tips positions. f, Time-dependence PINEM image intensity at the left and right tip in $\mathbf{b}$. The solid lines are Gaussian fits. The Gaussian fitted FWHM of the intensity profile is about 843 fs at the right tip and $786 \mathrm{fs}$ at the left tip. The optical pump wavelength is $1030 \mathrm{~nm}$ and the laser fluence is $1.3 \mathrm{~mJ} / \mathrm{cm}^{2}$. The scale bar in a-c represents $200 \mathrm{~nm}$. 

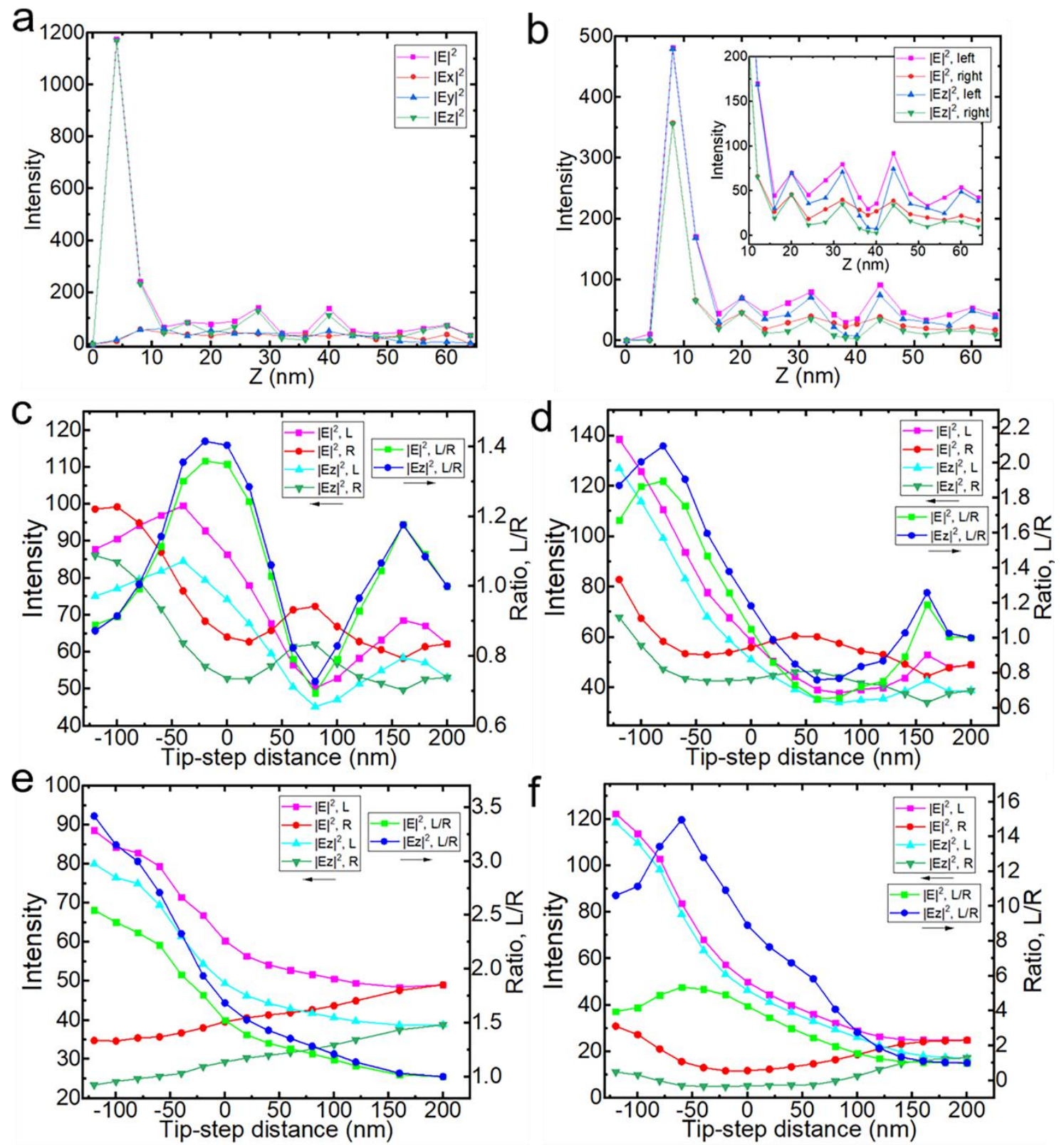

Figure S3. DDA simulations of graphene step effect on the plasmonic field of the GNC. a, The maximum intensity of plasmonic field at the left tip of the GNC in Fig.4a change in zdirection. $\mathbf{b}$, The maximum field intensities of total field $|\mathrm{E}|^{2}$ and $\mathrm{z}$-component field $\left|\mathrm{E}_{\mathrm{z}}\right|^{2}$ at the left and right tips of the GNC in Fig.4b change in z-direction. The insert in $\mathbf{b}$ shows the field intensity plotting magnified view for $\mathrm{z}>10 \mathrm{~nm}$. The maximum field intensity of total field $|\mathrm{E}|^{2}$ and z-component field $\left|E_{z}\right|^{2}$ at the left and right tips of the GNC change with tip-step distance using similar setup as Fig, 4b, but with different graphene substrate thickness and step height: c, $2 \mathrm{~nm}$ graphene substrate and $2 \mathrm{~nm}$ graphene step on it, d, $2 \mathrm{~nm}+4 \mathrm{~nm}, \mathbf{e} 4 \mathrm{~nm}+2 \mathrm{~nm}, \mathrm{f}, 4 \mathrm{~nm}+8$ $\mathrm{nm}$. 

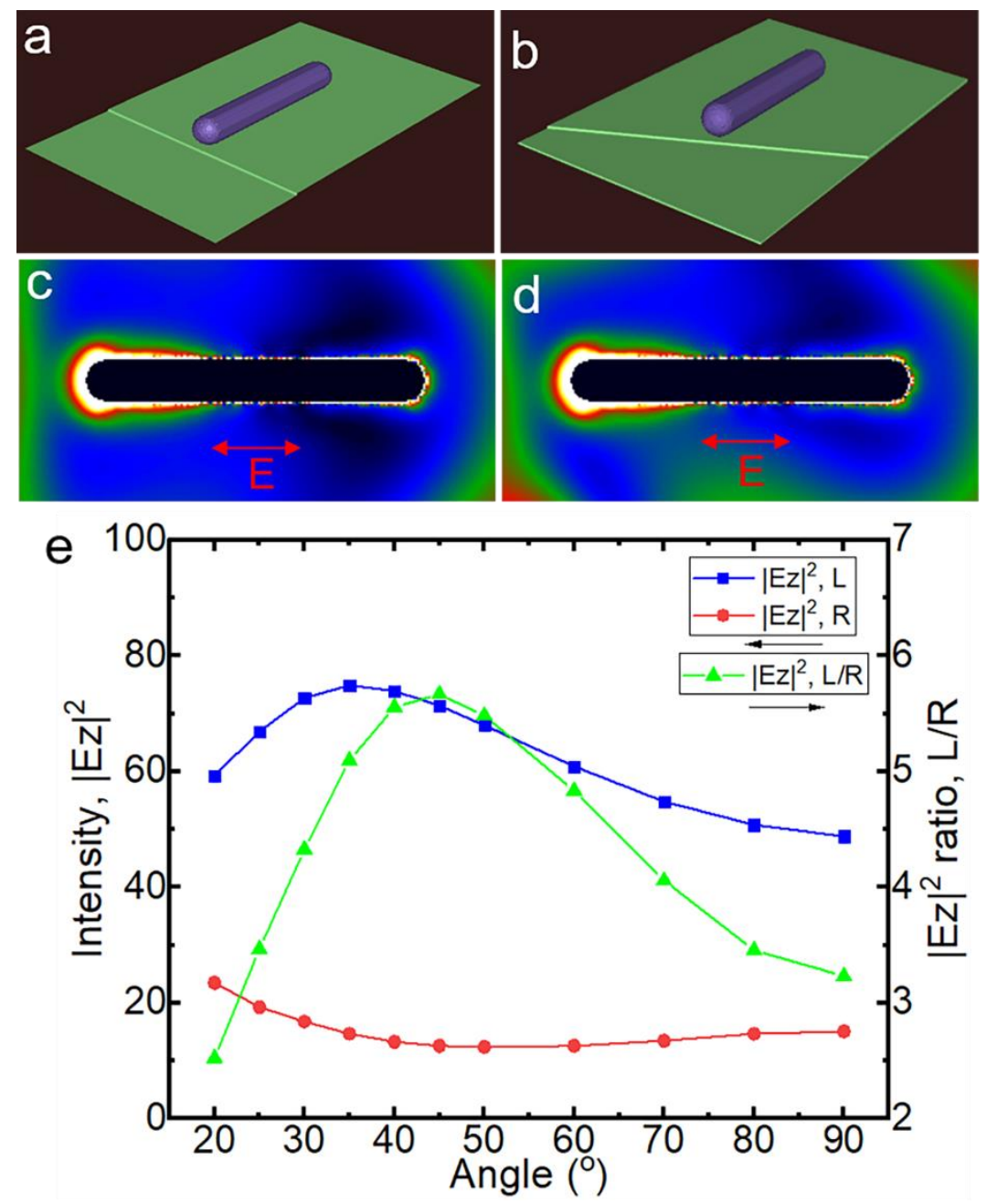

Figure S4 Angle effect on the tuning of plasmonic field of the GNC nearby a graphene step. $\mathbf{a}$ and $\mathbf{b}$ show two hybrid models of the GNC (60x360 nm) and graphene step (4 nm) on a graphene substrate of $4 \mathrm{~nm}$ with the angle of $90^{\circ}$ and $60^{\circ}$ between the long axis of the GNC and graphene step edge. $\mathbf{c}$ and $\mathbf{d}$ are the $\mathrm{z}$-component plasmonic field intensities distributions at plane height of $52 \mathrm{~nm}$ above top surface of the graphene for the angle of $90^{\circ}$ and $60^{\circ}$ under optical excitation of $1030 \mathrm{~nm}$ with light polarization parallel to the long axis of the GNC, respectively. $\mathbf{e}$ plots the maximum z-component plasmonic field intensities $\left|\mathrm{E}_{\mathrm{z}}\right|^{2}$ at the left tip and right tip and their ratio of L/R with changing of the angle between the long axis of the GNC and the graphene step edge. 

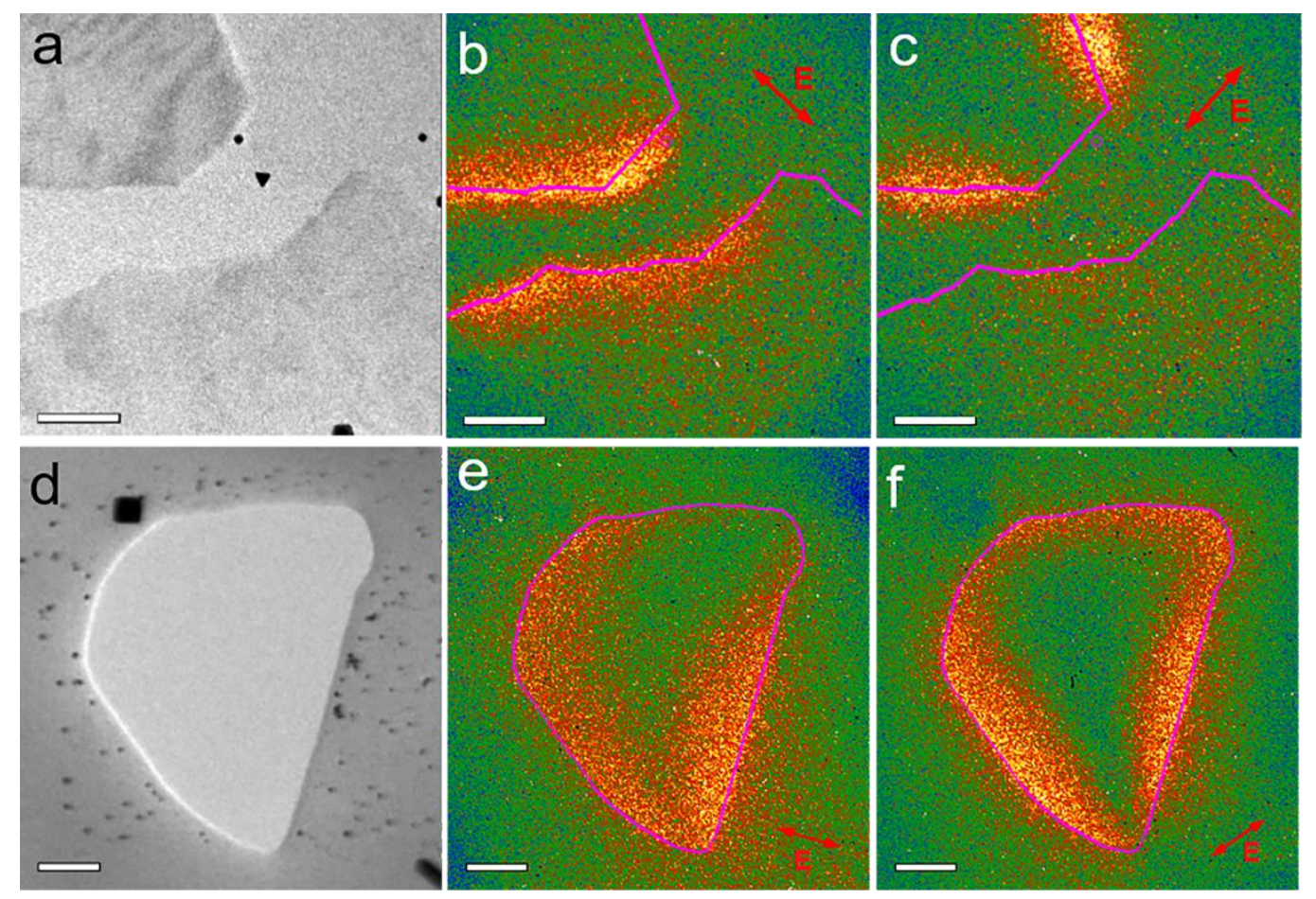

Figure S5 Plasmonic field mapping nearby a graphene step. a, TEM bright field image of the graphene with steps. The gap distance between the steps is from 200 to $300 \mathrm{~nm}$. b and c are PINEM images obtained at delay time of $t=0$ ps with different light polarizations indicated by the arrows. d, TEM bright field image of graphene with one irregular hole of about $835 \times 1200 \mathrm{~nm}$. e and $\mathbf{f}$ show PINEM images obtained at delay time of $\mathrm{t}=0 \mathrm{ps}$ with different light polarizations. The optical pump wavelength is $1030 \mathrm{~nm}$ and the laser fluence is $1.3 \mathrm{~mJ} / \mathrm{cm}^{2}$. The scale bar in the above images represents $200 \mathrm{~nm}$. The colored lines on the PINEM images indicate the step or edge position. 